\title{
RADIATIVE PROCESSES IN ATOMIC COLLISIONS AT STORAGE RINGS
}

\author{
L.R. Andersson* and J. Burgdörfer \\ Department of Physics and Astronomy, 401 A.H. Nielsen Bldg., \\ University of Tennessee, Knoxville, TN 37996-1200, USA \\ and Oak Ridge National Laboratory, Oak Ridge, TN 37931-6377, USA
}

\section{ABSTRACT}

We discuss radiative electron capture into excited states of fast, highly charged ions from neutral targets. Capture to high $n$ shells and to low-lying projectile continuum states is studied. We show that capture into excited states can account for up to $50 \%$ of the radiative capture cross section at the "Bremsstrahlung" t'.Jreshold. The feasibility of state-resolved measurements at storage rings is discussed.

\section{INTRODUCTION}

In collisions involving fast ions, emission of $\mathrm{X}$-rays due to various processes can occur. Besides the emission of characteristic X-ray lines originating in the relaxation of excited projectile or target states or $\gamma$-rays from nuclear transitions, continuous emission from transitions between transient molecular states (MO Xrays) and radiative electron capture (REC) will be present. The continuous REC and MO X-ray spectra are, apart from their intrinsic interest, important for analyzing the non-constant background of line-emission spectra. Since MO X-rays are most important in the intermediate and low velocity regime, we are concerned with $\mathrm{REC}$, which represents the dominant contribution to continuous $\mathrm{X}$-rays at high collision velocities.

$\mathrm{REC}$ is most conveniently visualized as a free-bound transition of a quasi-free target electron as seen in the projectile rest frame. The electron has a velocity distribution given by the Doppler-broadened momentum distribution of the initially bound target state ceniered around a mean velocity equal to the collision velocity. In addition to REC, processes where the final state of the electron is in the continuum of both projectile and target (usually termed radiative ionization (RI), radiative capture to the continuum (RCC), or atomic bremsstrahlung) are possible. The latter is analogous to the free-free ta ansition for atomic bremsstrahlung when one approximates the binding to the initial target state by the Galilei-shifted momentum distribution. We will in the following denote this process by RCC in order to stress the continuity across the ionization limit when the final state is a low-lying continuum state in the frame of the projectile. One objective of the present work is to explore the REC and RCC processes in the vicinity of the "bremsstrahlung threshold" corresponding to transitions to projectile states close to the continuum limit.

- Present address: Manne Siegbahn Institute of Physics, S-10405 Stockholm, Sweden 
REC was experimentally first observed by Schnopper et al. ${ }^{1}$ in collisions between heavy ions and solid targets. In addition to the broad X-ray distribution due to RCC and characteristic X-ray lines, a pcak deriving from capture to the $\mathrm{K}$ shell of the projectile (K-REC) was obscrved. Considerable experimental ${ }^{2-5}$ and theoretical ${ }^{6-8}$ work followed, mainly concentrating on K-REC since this is the dominant capture channel at high velocities and its spectral signature can be relatively easily separated from other features. Only very recently, studies of REC into excited states have become available. ${ }^{9-12}$ REC into excited shells of the projectile is difficult to measure unless the parameters of the collision system are chosen carefully.

We present in the following REC and $\mathrm{RCC}$ spectra in $\mathrm{Ne}^{10+}-\mathrm{H}$ collisions at a relatively moderate collision velocily calculated in the impulse approximation, in order to analyze how the REC spectrum for high- $n$ capture and the low-continuum RCC spectrum build up the total X-ray spectrum. We, furthermore, illustrate the utility of storage rings for shell-resolved REC experiments. We also stress the interrelation to radiative recombination ( $R R)$.

Experimental studies of radiative recombination (RR) have been carried out in single-pass experiments in $\AA \cdot \mathrm{rus}^{13}$ and in the storage rings in Heidelberg ${ }^{14}$ and Stockholm ${ }^{15}$ using the electron cooler beam as the target. It is thus interesting to note that the two related atomic processes can be explored in detail in completely different velocity regimes with these new experimental facilities.

\section{CRITERION FOR SHELL RESOLVED REC SPECTRA}

We want to establish a simple criterion under which REC peaks resulting from capture into the $n^{\text {th }}$ and $n+1^{\text {th }}$ shell of a hydrogenic projectile can be resolved. The key is that the width of the IREC peaks, originating in the width of the target electron velocity distribution and the Doppler broadening when transformed to the projectile rest frame, is intrinsic and limits the ability to resolve adjacent peaks. Denoting the width of the initial target-electron velocity distribution by $\Gamma$ (on a velocity scale in a.u.), the corresponding width of the energy distribution associated with the Doppler shifted velocity distribution is $\approx 2 v \Gamma$. Consequently, for bare projectiles, a criterion for resolving the adjacent peaks resulting from capture into the $n$ and $n+1$ shells can be written as

$$
Z_{P}^{2} \frac{2 n+1}{n^{2}(n+1)^{2}}>2 v \Gamma,
$$

here $Z_{P}$ is the projectile charge. For an atomic target, $\Gamma$ is of the order of the (effective) charge $Z_{T}$. For conduction band electrons of a solid target, $\Gamma$ is of the order of the Fermi velocity $v_{F}$. Weakly bound electrons with small $\Gamma$ (or $Z_{T}$ ) are thercfore preferable.

A second intrinsic difficulty associated with state-resolved REC spectra is the simultaneous presence of characteristic target and projectile $X$-ray lines. In order to separate the line spectrum from the REC spectrum, the projectile and target 
Sommerfeld parameters should not exceed unity. (This is also the parameter regime where perturbative models are expected to give the most reliable results.) Since we consider systems with $Z_{P}>Z_{T}$, the condition for a REC spectrum unperturbed by $\mathrm{X}$-ray lines is simply

$$
\frac{Z_{P}}{v}<1 .
$$

Using scaled variables $Z_{p} / \Gamma$ and $v / \Gamma$, the criteria (Eqs. (1) and (2)) can be representcd in one diagram (Fig. 1). Eq. (1) results in a family of curves each signifying the resolution of the $n^{\text {th }}$ and $n+1^{\text {th }}$ peak $(n=1,2 \ldots)$ while Eq. (2) represents the $45^{\circ}$ line indicating that only the parameter space corresponding to the lower half of the diagram admits X-ray spectra unperturbed from characieristic lines. We show in Fig. 1 also the parameter range of recent REC experiments using gas targets (open symbols) and solid targets (solid symbols). As can be seen, most experiments which satisficd (1) for $n \geq 2$ did not satisfy Eq. (2). The regime with high $Z_{P}$ and high $v$, but small $Z_{P} / v$ is, to a large extent, unexplored, the obvious

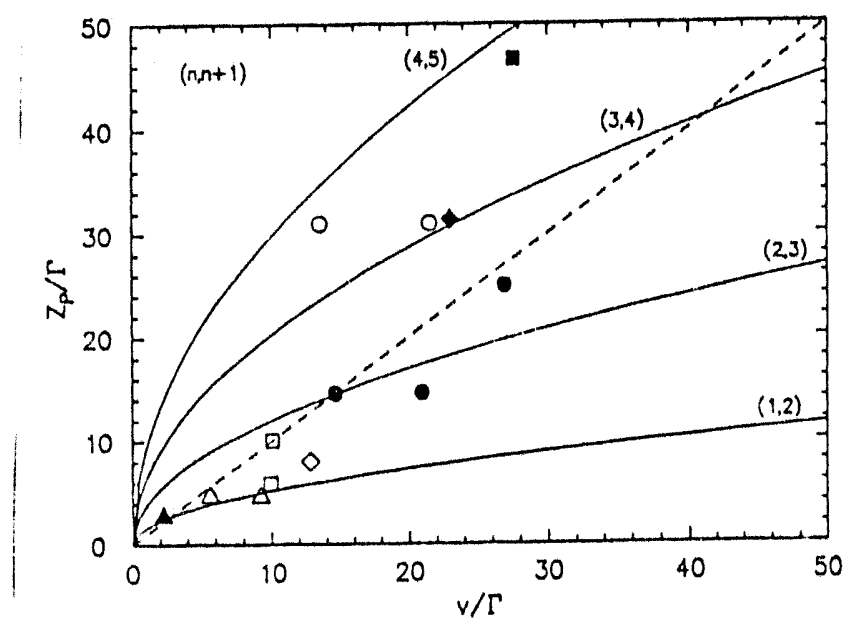

Figure 1: Criteria for well-resolved peaks for REC into the $n$ and $n+1$ shells of bare projectiles with charge $Z_{p}, \Gamma$ denotes the width of the velocity distribution of the target electron. Symbols represent the region marked by experiments. $\square$ : Kienle et al. ${ }^{2} ; \diamond$ : Schnopper et al. ${ }^{3} ; \triangle$ : Tawara et al. ${ }^{4} ; 0$ : Stöhllker et al. ${ }^{12} ; 0$ : Anholt et al. ${ }^{10} ;$-: Andriamonje $e t$ al. ${ }^{11} ; \mathbf{\Delta}$ : Jakubaßa $e t$ al. ${ }^{16} ; \bullet$ : Datz et al. ${ }^{17}$ Open and solid symbols denote gas and solid targets, respectively.

reason being the smill cross section. In Fig. 2 is shown schematically the contours of constant cross suction plotted against the reduced parameters $Z_{P} / \Gamma$ and $v / \Gamma$. For simplicity we have used the recombination cross section for free electrons which is a function of $Z_{P} / v$ only.

The main reason for the frequent use of a solid target is, of course, the high density of target electrons which improves the count rates counteracting the small cross section. Solid targets introduce, however, other difficulties when making 


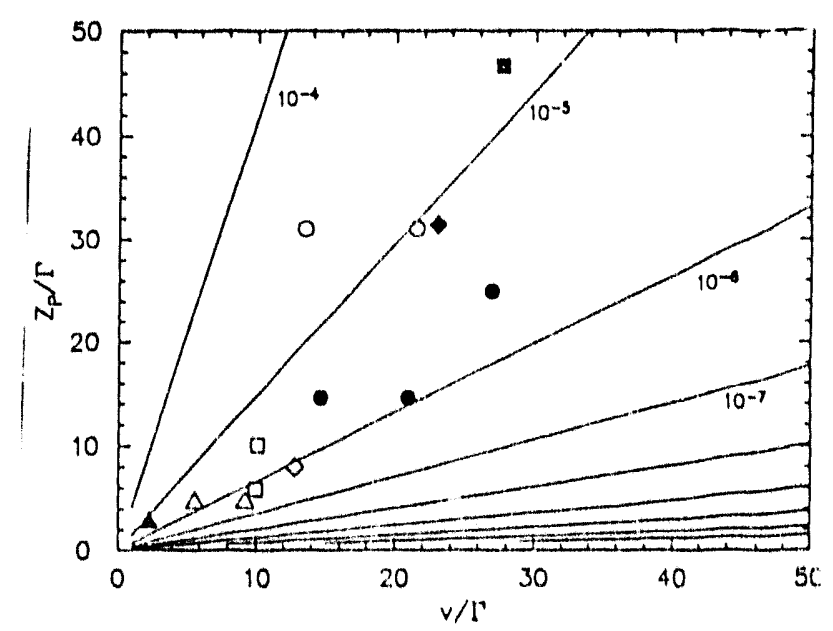

Figure 2: Contour lines of the K-shell radiative recombination cross section in the $Z_{p} / \Gamma-v / \Gamma$ plane. The increment between adjacent contours is one order of magnitude. The cross section in a.u. is indicated at a few contours. Symbols as in Fig. 1.

detailed comparison with theory. Secondary processes producing $X$-rays, such as secondary bremsstrahlung, ${ }^{10}$ will contribute an additional background which can, in unfavorable cases, completely overtake the REC spectrum. Further, unless the projectile ions are channeled, the charge state of the projectile is ill-defined. Under channeling conditions, the equilibrium charge state is close to the initial charge state, and, in addition, $\Gamma$ can be reduced so that REC into excited states can be measured. ${ }^{11}$

In the regime of small $Z_{P} / v$ heavy-ion storage rings appear to be the most promising candidates for detailed studies of REC in last collisions. ${ }^{12}$ The high luminosity of the circulating beam compensates for the low target density and small cross sections. A caveat is, however, that a much larger mechanical capture cross section must not lead to an alteration of the initial charge state of the beam. It should be noted that $\Gamma$ is greatly reduced if a free-electron target is used. Fig. I therefore indicates that radiative recombination measurements ${ }^{13-15}$ using the electron cooler bean as a target should permit the resolution of high $n$ states. In this case $Z_{p} / \Gamma>>1$ and $v / \Gamma \approx 1$ corresponding to the upper left corner of the parameter space of libit. 1 .

\section{THEORY}

The simple picture for $R E C$ as a radiative-recombination event in the rest frame of the projectile with the velocity distribution of the incident electron given by the properly transformed momentum distribution of the target bound state leads to the impulse approximation (IA). ${ }^{6,7}$ The cross section differential in the photon energy and emission solid angle for capture to the final projectile state $f$ 
is given by

$$
\begin{aligned}
\frac{d \sigma_{\rho}^{R E C}}{d \omega d \Omega} & =\sum_{\lambda} \frac{4 \pi^{2} \omega}{c^{3} v} \int d^{3} q\left|\phi_{i}^{T}(\mathrm{q}+\mathrm{v})\right|^{2}\left|\left\langle\psi_{\rho}^{P}\left|\mathrm{e}_{\lambda} \cdot \mathrm{p}\right| \psi_{\mathrm{q}}^{P}\right\rangle\right|^{2} \\
& \times \delta\left(E_{\rho}^{P}-E_{i}^{T}+\omega+v^{2} / 2+\mathrm{q} \cdot \mathrm{v}\right),
\end{aligned}
$$

where $\omega$ is the photon energy, $c$ is the speed of light, $e_{\lambda}$ are the polarization vectors oi the photon, $v$ is the collision velocity, $E_{f}^{P}$ and $E_{i}^{P}$ are the (negative) binding energies of the final and initial states of the electron, respectively, $\phi_{i}^{T}$ is the momentum distribution of the initial target state, $\psi_{\rho}^{P}$ is the final bound state on the projectile, and $\psi_{\eta}^{P}$ is a projectile continuum state of momentum q.

Correspondingly, the cross section for $\mathrm{RCC}$ is obtained by replacing $\psi_{f}^{P}$ by a final state in the projectile continuum $\psi_{\mathrm{k}}^{\mathrm{P}} 8,18$

$$
\begin{aligned}
\frac{d \sigma^{R C C}}{d \omega d \Omega d^{3} k} & =\sum_{\lambda} \frac{4 \pi^{2} \omega}{c^{3} v} \int d^{3} q\left|\phi_{i}^{T}(\mathrm{q}+\mathrm{v})\right|^{2}\left|\left\langle\psi_{\mathrm{k}}^{P}\left|\mathbf{e}_{\lambda} \cdot \mathrm{p}\right| \psi_{\mathrm{q}}^{P}\right\rangle\right|^{2} \\
& \times \delta\left(k^{2} / 2-E_{\mathrm{i}}^{T}+\omega+v^{2} / 2+\mathrm{q} \cdot \mathrm{v}\right)
\end{aligned}
$$

where $k$ is the final momentum of the electron in the projectile rest frame. The above expressions for the REC and RCC cross sections are on-shell approximations to the distorted strong potential Born (DSPB) expressions, ${ }^{19}$ in which the state $\psi_{q}^{P}$ is off the energy shell since, strictly, the target electron does not propagate with energy $q^{2} / 2$ in the projectile rest frame. For radiative electron capture, differences between the IA and the DSPB are expected to be small. ${ }^{18,19}$

In (3) and (4) the emission angles and energies of the photon are given in the projectile rest frame. The Lorentz transformation to laboratory quantities is given by

$$
\begin{aligned}
\cos \theta_{l a b} & =\frac{\cos \theta+\beta}{1+\beta \cos \theta} \\
\omega_{l a b} & =\omega \gamma^{-1}\left(1-\beta \cos \theta_{l a b}\right)^{-1} \\
\frac{d \sigma}{d \omega_{l a b} d \Omega_{l a b}} & =\gamma^{-1}\left(1-\beta \cos \theta_{l a b}\right)^{-1} \frac{d \sigma}{d \omega d \Omega}
\end{aligned}
$$

where $\beta=v / c, \gamma^{-2}=1-\beta^{2}$, and the angles are taken with respect to the direction of the projectile velocity.

We restrict ourselves to fully' stripped projectiles, for which dipole matrix elements are avalable in closed form. For free-bound transitions, the dipole matrix elements have been evaluated by Stubbe ${ }^{20}$ and for the free-free transitions by Sommerfold." Assuming further that the target momentum distribution is spherically symmetric and that the magnetic quantum number $m$ of the final state is not observed, one-dimensional quadratures are required in order to evaluate (3) and (t). Notice that in order to obtain $d \sigma^{R C C} /(d \omega d \Omega)$ two additional numerical quadratures are necessary. Sommerfeld's ingenious method to integrate out both 


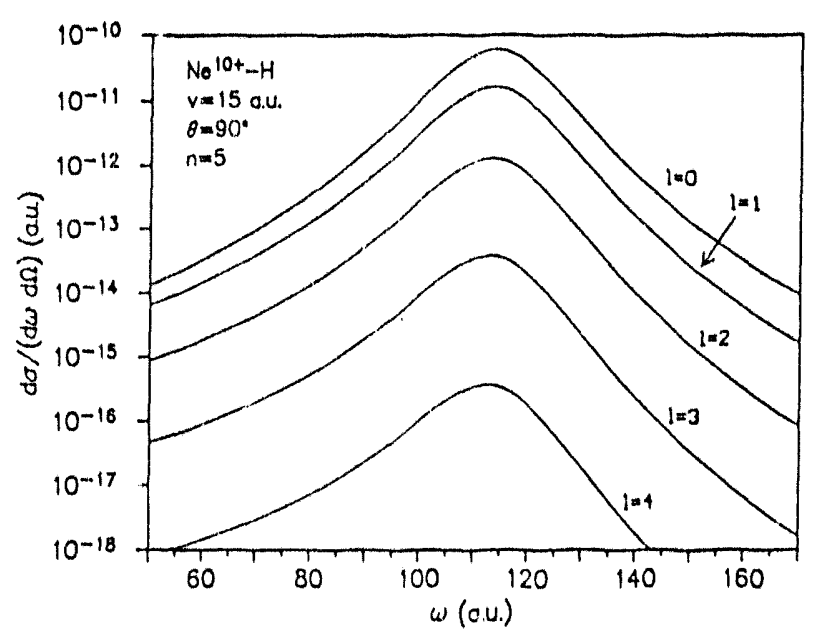

Figure 3: REC cross section for $\mathrm{Ne}^{10+}-\mathrm{H}$ collisions at $v=15$ a.u. The photon emission angle is $90^{\circ}$ in the projectile frame. The curves represent capture into various $l$-substates of the $n=5$ shell of $\mathrm{Ne}^{9+}$.

the photon and electron emission angles ${ }^{21}$ can only be used for calculating the singly differential cross section $d \sigma^{R C C} / d \omega$.

\section{RESULTS AND DISCUSSION}

We present here results for REC and RCC for the collision system $\mathrm{Ne}^{10+}-\mathrm{H}$ at $v=15$ a.u. $(E=5.6 \mathrm{MeV} / \mathrm{amu})$. In Fig. 3 is shown the cross section for capture into $n=5$ broken down into the various $l$-states. The low angular momenta dominate completely, with $l=0$ and $l=1$ accounting for $99 \%$ of the cross section. This is in sharp contrast to $R R$ at low energies where the l-distribution peaks at $l / n \approx 0.3$ for high $n .^{22}$

In Fig. 4 is shown the REC-spectrum for $n \leq 10$ summed over all $/$ states for the same collision system. The dashed line indicates the 'RCC.threshold', i.e. the position $\omega_{C}$ of the centroid of the REC peak corresponding to capture into states with $n \rightarrow \infty$. Its value is given by

$$
\omega_{C}=v^{2} / 2+E_{i}^{T}
$$

Since $Z_{P} / v=2 / 3$ the $N$ X $X$-ray lines are well below $\omega_{C}$ and thus separated from the REC spectrum.

The height of the REC-peaks rapidly approach an $n^{-3}$ dependence with increasing $n$. However, since the density of bound projectile states close to the $R C C^{\prime}$-threshold scale as $n^{3}$, the high-n peaks will provide a finite contribution to the total X-ray spectrum. In order to study the behavior of the spectrum close to $\omega=\omega_{C}$, the RCC cross section must be included as well. The frec-electron bremsstrahlung spectrum has a strict upper cut-off at $\omega^{\prime}$, corresponding to the slowing down of the electron to rest with respect to the projectile. With the finite 


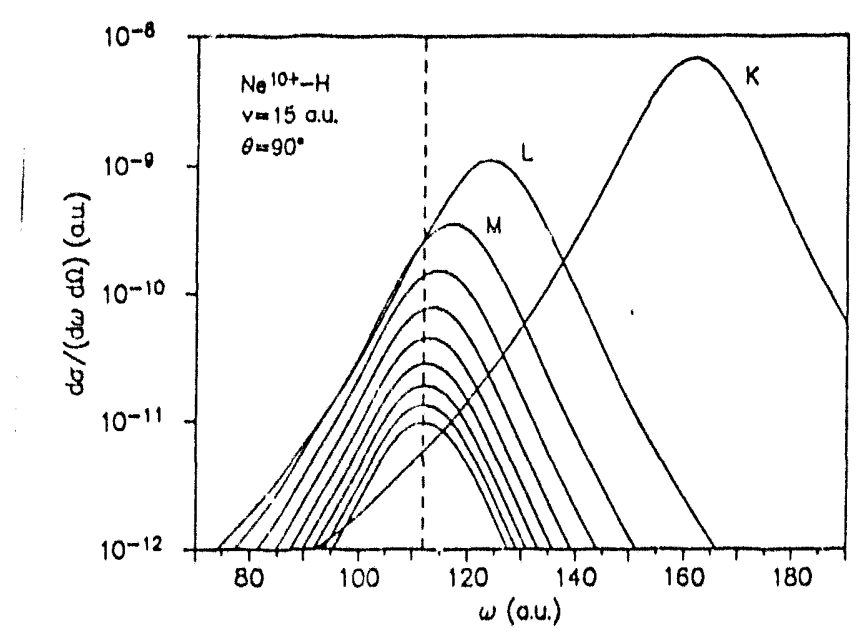

Figure 4: REC cross sections for capture into the 10 lowest lying principal shells of $\mathrm{Ne}^{9+}$ in $\mathrm{Ne}^{10+} \ldots$ II collisions at $v=15$ a.u. The photon emission angle is $90^{\circ}$ in the projectile frame. 'The dashed line indicates the position of $\omega_{C}$ (see Eq. (6)).

width of the velocity distribution of the initial state the RCC spectrum extends to frequencies above $\omega_{C}$. In Fig. 5 is shown the REC and RCC spectra and their sum. Shells up to $n=50$ were included which was necessary in order to obtain convergence. In Fig. 6 is illustrated the convergence of the spectrum as a function

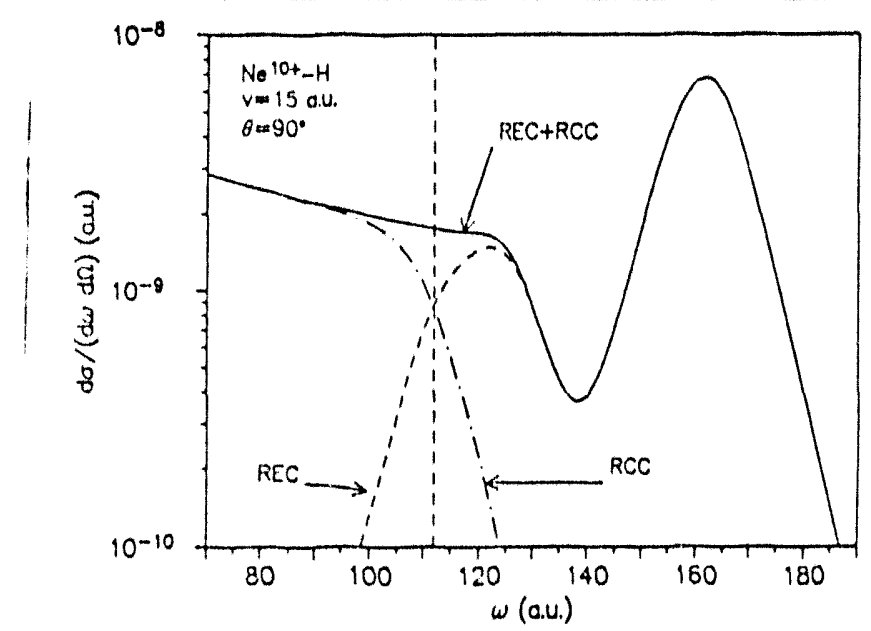

Figure 5: REC and RCC cross sections for $\mathrm{Ne}^{10+}-\mathrm{H}$ collisions at $v=15$ a.u. The photon observation angle is $90^{\circ}$ in the projectile frame.

of the maximum $n$ included. One remarkable observation is that the shells with $2<n \leq 50$ contribute about $50 \%$ to the total cross section in the vicinity of $\omega_{C}$.

All the above calculations were performed assuming that the dipole approximation is valid. The wave length of a photon equals the hydrogenic Bohr radius at $\omega \approx 4 \mathrm{keV}$, which is close to the $K$-REC peak energy for the example studied 


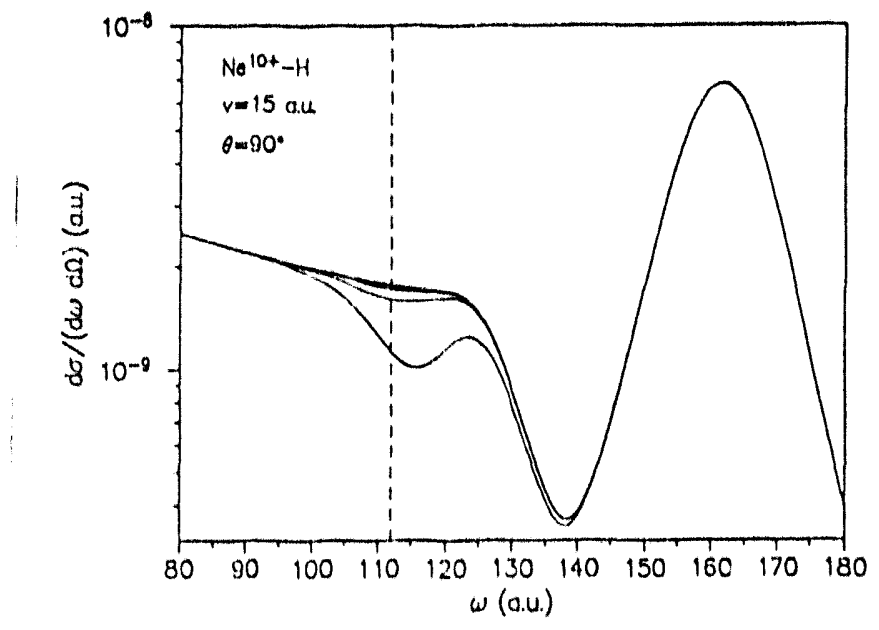

Figure 6: Total REC and RCC spectrum for $\mathrm{Ne}^{10+}$. II collisions at $v=15$ a.u. The different curves represent the spectrum obtained by summing the RLC final states up to various maximum $n$. From below, the curves correspond to $n_{\max }=2,5,10$, and 50 , respectively.

above. For higher projectile charges and/or velocities the photon energies will exceed this value and retardation effects will become increasingly important. The main effect of retardation is to shift the photon angular distribution to backward angles in the projectile frame. For the case of free incident electrons captured into the 1 s state the cross section in the projectile frame (when integrated over photon encrgies) has the angular dependence

$$
\frac{d \sigma^{R E C}}{d \Omega} \times \frac{\sin ^{2} \theta}{(1+\beta \cos \theta)^{4}} .
$$

After the Lorentz transformation to the laboratory system the angular distribution is proportional to $\sin ^{2} \theta_{\text {lub }}$, which is equivalent to the angular distribution in the projectile frame withont retardation. This cancellation between retardation and Lorentz transformat..n was experimentally observed by Spindler et al. ${ }^{4}$ who explained it based on the free electron angular distributions. Three-body calculations by Pacher it al. ${ }^{23}$ show that the K-REC cross sections are, indeed, symmetric with respect to the perpendicular photon-emission direction to a good degree of approximation when retardation is included and the projectile velocity is not approaching the relativistic region $(u<c / 2)$. We find the same result for K-REC using the LA. However, for L-REC the cancellation effect is not complete for individual $l$ states. Nevertheless, the sum of capture into $2 s$ and $2 p$ is, indeed, symmetric with respect to $0=90^{\circ}$. This is a direct consequence of the Fock sum rule for the momentum space wavefunctions of hydrogenic ions,

$$
\sum_{\ln }\left|b_{n ! m}(q)\right|^{2}=n^{2}\left|\phi_{1 s}(q)\right|_{z=z_{1} / n}^{2}
$$


i.e., the sum over the momentum distributions over all $l$ for a fixed $n$ is equivalent to a $1 \mathrm{~s}$ momentum distribution with an effective charge. This sum rule appears to be applicable to a good degree of approximation even when the full IA is used.

The X-ray spectrum (i.e. $d r /(d \omega d \Omega)$ ) as a function of the photon emission angle can be used for indirectly determining capture into individual $l$ states. In our example, the $2 p$ content of the L-REC peak is $38 \%, 18 \%$, and $42 \%$ at the laboratory emission angles 30,90 , and 150 degrees, respectively. The backwardforward asymmetry of the ratio of the K- and L-REC peaks is therefore more pronounced than for a pure $2 s$ final state.

\section{CONCLUSIONS}

We have shown how radiative electron capture into bound and continuum projectile states builds up the total X-ray spectrum. In order to properly represent the spectrum close to the continuum limit of the projectile spectrum, REC into a relatively large number of bound projectile shells must be included. We have discussed criteria for selecting the parameters of the collision system in order to make detailed comparisons between experiments and theory. Heavy-ion storage rings seem to be well suited for this purpose.

\section{REFERENCES}

${ }^{1}$ H.W. Schnopper, H.D. Betz, J.P. Delvaille, K. Kalata, A.R. Sohlval, K.W. Jones, and H.E. Wegner, Phys. Rev. Lett. 29, 898 (1972).

2 P. Kienle, M. Kleber, B. Povh, R.M. Diamond, F.S. Stephens, E. Grosse, M.R. Maier, and D. Proetel, Phys. Rev. Lett. 31, 1099 (1973).

${ }^{3}$ H.W. Schnopper, J.P. Delvaille, K. Kalata, A.R. Sohlval, M. Abdulwahab, K.W. Jones, and H.E. Wegner, Phys. Lett. 47A, 61 (1974).

${ }^{4}$ E. Spindler, H.-D. Betz, and F. Bell, Phys. Rev. Lett. 42, 832 (1979).

${ }^{5}$ H. Tawara, P. Richard, and K. Kawatsura, Phys. Rev. A 26, 154 (1982).

${ }^{6}$ J.S. Briggs and K. Dettmann, Phys. Rev. Lett. 33, 1123 (1974).

${ }^{7}$ M. Kluber and D.H. Jakubaßa, Nucl. Phys. A252, 152 (1975).

${ }^{8}$ D.H. Jakubaßa and M. Kleber, Z. Phys. A 273, 29 (1975).

9 J.E. Miraglia, C.R. Garibotti, and A.D. González, Phy's. Rev. A 32, 250 (1985).

10 R. Anholt, Ch. Stoller, J.D. Molitoris, D.W. Spooner, E. Morenzoni, S.A. Andriamonje, W.E. Meyerhof, H. Bowman, J.S. X̌u, Z.-Z. Xu, J.O. Rasmussen, and D.H.H. Hoffmann, Phy's. Rev. A 33, 2270 (1986).

$"$ S. Andriamonje, M. Chevalier, C. Cohen, J. Dural, M.J. Gaillard, R. Genre, M. Hage-Ali, R. Kirsch, A. L'Hoir, B. Mazuy, J. Mory, J. Moulin, J.C. Poizat, J. Remillieux, D. Schmaus, and M. Toulemonde, Phys. Rev. Lett. 59, 2271 (1087).

12 Th. Stöhlker, C. Kozhuharov, A.E. Livingston, P.H. Mokler, Z. Stachura, and A. Warczak, Z. Phy's. D 23, 121 (1992).

13 L.LI. Andersen, G.-Y. Pan, and II.T. Schmidt, J. Phys. B: At. Mol. Opt. Phys. 25, $277(1992)$. 
14 A. Wolf, J. Berger, M. Bock, D. Habs, B. Hochadel, G. Kilgus, G. Neureither, U. Schramm, D. Schwalm, E. Szmola, A. Müller, M. Wagner, and R. Schuch, Z. Phys. D 21, S69 (1991).

15 R. Schuch, T. Quinteros, M. Pajek, Y. Haruyama, H. Danared, G. Hui, G. Andler, D. Schneider, and J. Starker, (in press) (1.993).

16 D.H. Jakubaßa-Amundsen, R. Höppler, and H.-D. Betz, J. Phys. B: At. Mol. Phys. 17, 3943 (1984).

17 S. Datz, P.F. Dittner, J. Gomez del Campo, H.F. Krause, T.M. Rosseel, and C.R. Vane, Z. Phys. D 21, 545 (1991).

18 D.H. Jakubaßa-Amundsen, J. Pliys. B: At. Mol. Phys. 20, 325 (1987).

19 K. Taulbjerg, R.O. Barrachina, and J.H. Macek, Phys. Rev. A 41, 207 (1990).

20 M. Stobbe, Ann. Phy's. (Leipzig) 7, 661 (1930).

21 A. Sommerfeld, Atombau und Spektrallinien 2. Auf., Bd. 2 (Braunschwcig: Vieweg \& Sohn) (1939).

22 M. Pajek and R. Schuch, Phys. Rev. A 45, 7894 (1:i22).

23 M.C. Pacher, A.D. González, and J.E. Miraglia, Phys. Rev. A 35, 4108 (1987).

\title{
DISCLAIMER
}

\begin{abstract}
This report was prepared as an account of work sponsored by an agency of the Unitod States Government. Neither the United States Government nor any agency thereof, nor any of their employees, makes any warranty, express or implied, or assumes any legal liability or responsibility for the accuracy, completeness, or usefulness of any information, apparatus, product, of process disclosed, or represent? that its use would not infringe privately owned rights. Reference herein to any specific commercial product, process, or service by trade name, trademark, manufacturer, or otherwise does not necessarily constitute or imply its endorsement, recommendation, or favoring by the United States Government or any agency thereof. The views and opinions of authors expressed herein do not necessarily state or reflect those of the United States Government or any agency thereof.
\end{abstract}



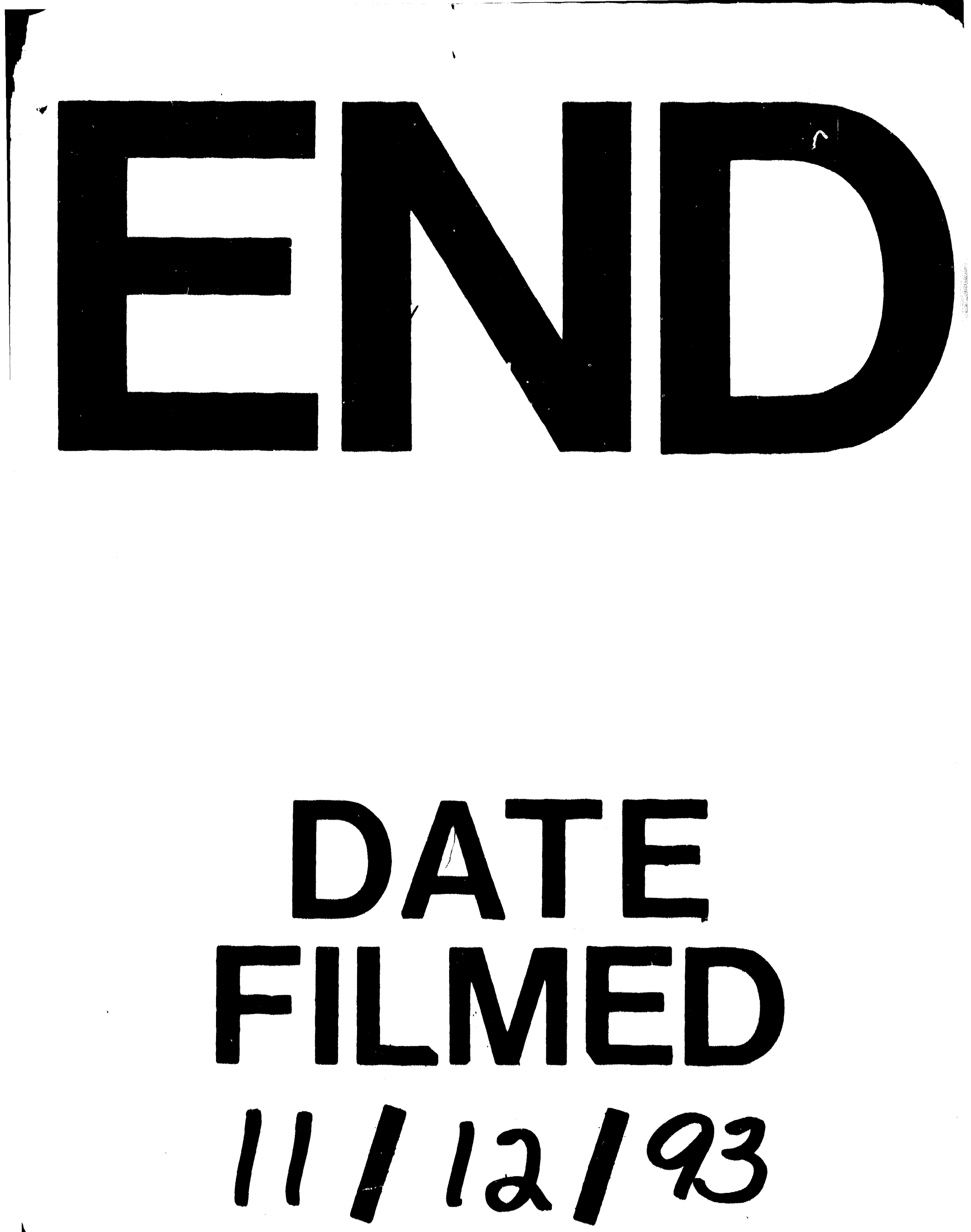


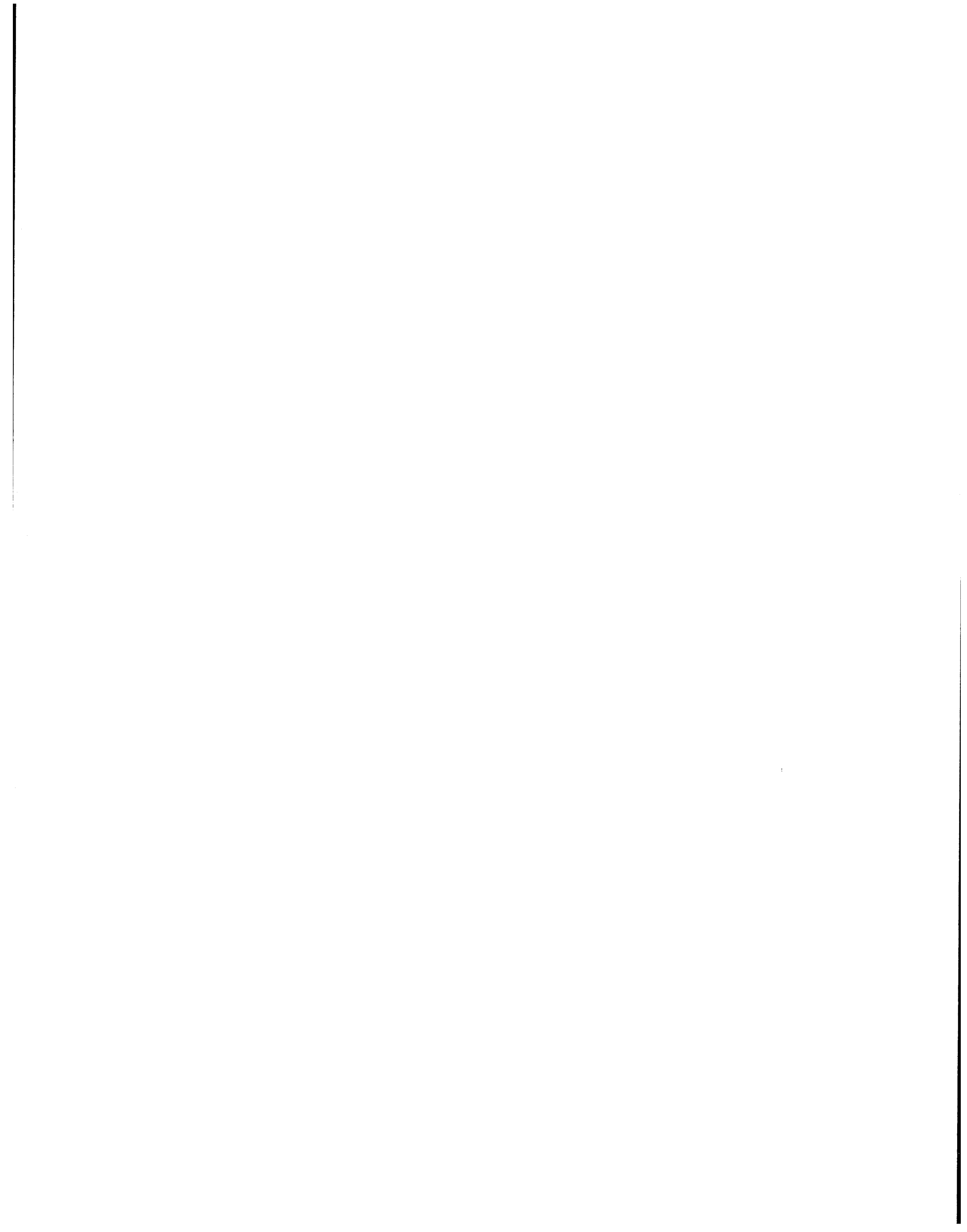

